\title{
IN VITRO STUDIES ON POSSIBLE SYNERGISTIC ACTION BETWEEN PENICILLIN AND BACITRACIN ${ }^{1}$
}

\author{
BY MARVIN C. BACHMAN \\ (From the Research and Development Division, Commercial Solvents Corporation, \\ Terre Haute, Indiana)
}

\section{INTRODUCTION}

A number of publications have appeared describing the successful use of mixtures of two or more antibacterial substances. In many instances the combined effect has indicated synergistic activity instead of being merely additive.

There are a number of reasons why it may be desirable to use these combinations of antibacterial substances. Resistance to one antibiotic is probably inherited independently to a second one. Thus when one or two cells per million are resistant to antibiotic $A$, the chances that they are also resistant to antibiotic $B$ are very low. When antibiotics A and B are used together, each in a concentration which will inhibit all but the resistant individuals, there will be no survivors, except in tremendous populations. Klein and Kimmelman found that the development of streptomycinresistant variants in vitro could be greatly reduced by the addition of small amounts of sulphadiazine. Furthermore, they found that subinhibitory concentrations of penicillin, streptomycin, and sulphadiazine, when combined, were highly effective in inhibiting bacterial multiplication and completely prevented the development of resistant variants. Their data indicate direct correlation between the inhibition of resistant variants and synergistic action with penicillin, streptomycin and sulphadiazine.

Recently Eagle and Fleischman described the synergistic action of bacitracin and penicillin against Treponema pallidum in rabbits. They found that when a penicillin and bacitracin mixture was used, the therapeutic levels of each antibiotic were significantly reduced.

\footnotetext{
1 Presented at the Second National Symposium on Recent Advances in Antibiotics Research held in Washington, D. C., April 11-12, 1949, under the auspices of the Antibiotics Study Section, National Institutes of Health, Public Health Service, Federal Security Agency.
}

There are many strains of Streptococcus, particularly of the viridans and enterococcus groups, which are refractory to penicillin, but susceptible to bacitracin. However, the toxicity of the present bacitracin seriously limits its parenteral use. It seemed worthwhile, then, to study the in vitro effect of penicillin and bacitracin combinations against these streptococci, keeping the bacitracin content of the mixture low because of its toxicity. Several of the Streptococcus strains used were recently isolated from cases of subacute bacterial endocarditis.

\section{PROCEDURE}

Our test procedure was as follows: Ten $\mathrm{ml}$ of filtered tryptose or nutrient broth, containing penicillin and bacitracin, both singly and in combination, were added to calibrated $6 \times \%$ inch tubes. The inoculum consisted of a saline suspension of cells washed from a 24-hour tryptose agar slant of the test organism. This inoculum was standardized and $0.1 \mathrm{ml}$ (about $10,000,000$ cells) was transferred to each tube of medium. The tubes were then incubated at $37^{\circ} \mathrm{C}$. Turbidity determinations were made at intervals over a 24-hour period, using a Lumetron colorimeter. The amounts of growth, expressed as per cent light transmission, were plotted against the hours of incubation.

\section{RESULTS}

A total of 18 strains of Streptococcus were used. Data obtained on four of them are presented in Figures 1-4.

\section{SUMMARY}

A study has been made of the in vitro effect of penicillin and bacitracin mixtures against several strains of $\alpha$ - and $\gamma$-hemolytic streptococci. Data obtained indicate synergistic action between the two antibiotics instead of merely an additive effect. The synergistic effect varies from strain to strain, being very pronounced with some cultures and less dramatic with others. 

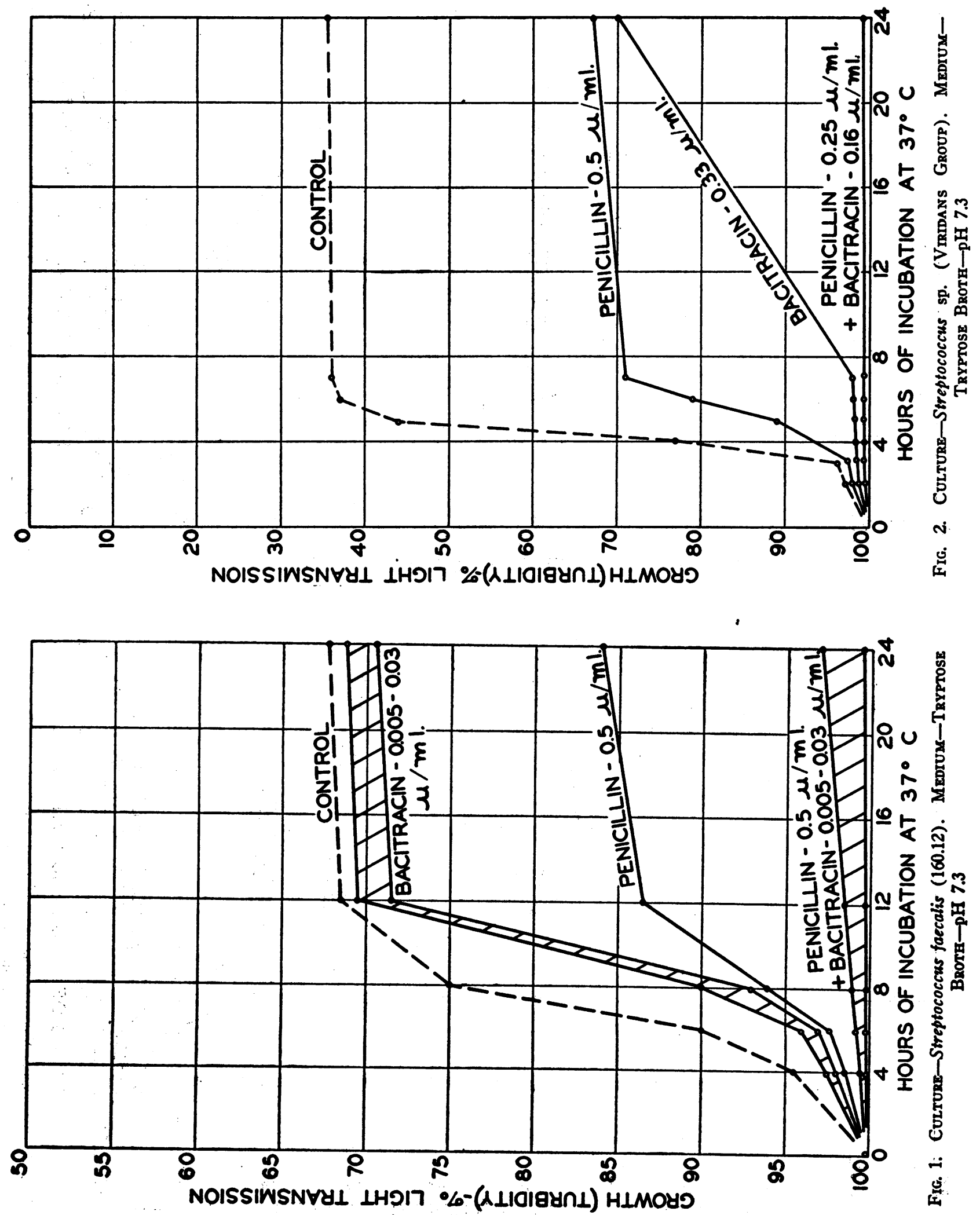

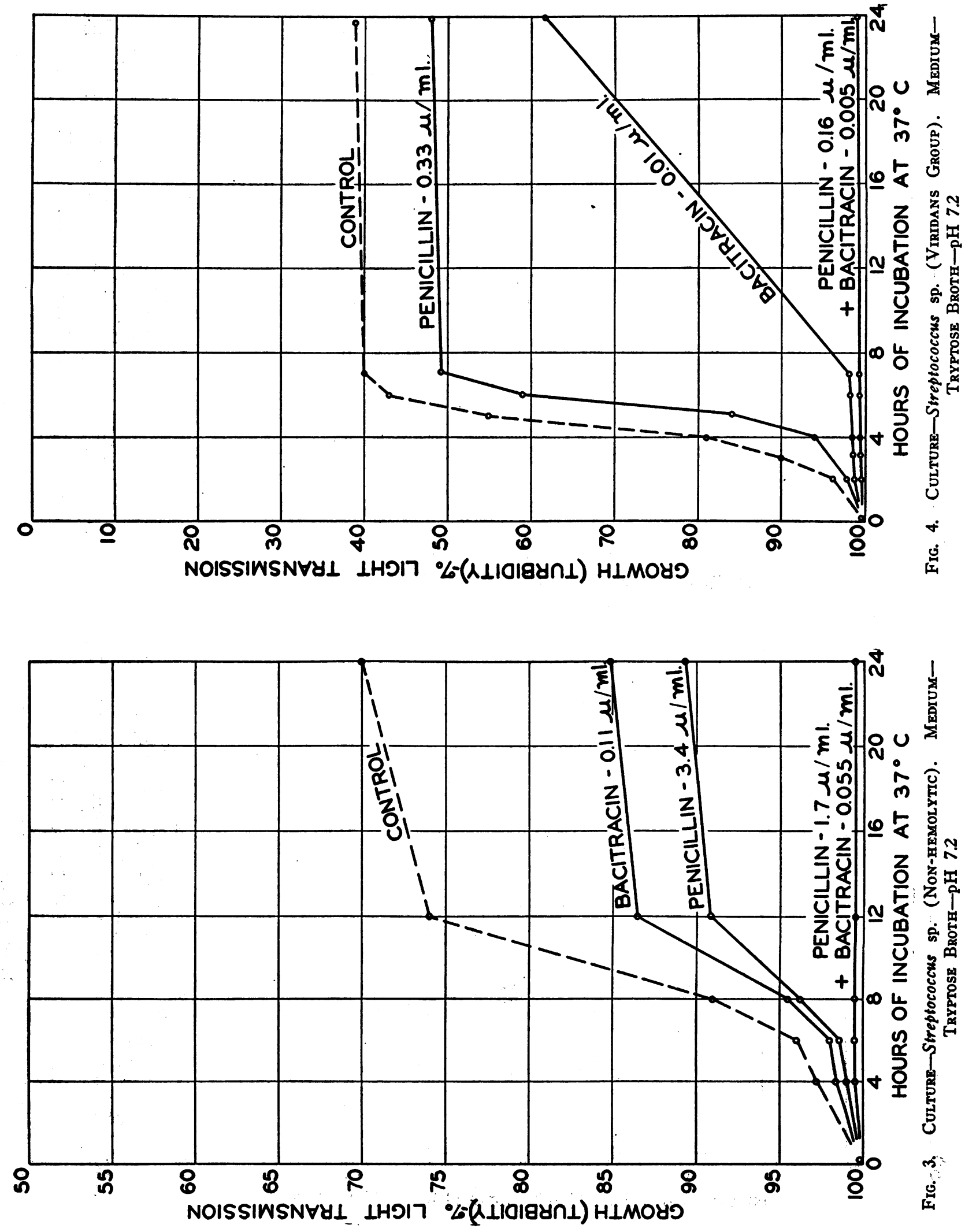